Akdemir G, et al. RMD Open. 2018;4

Atsumi T, et al. Ann Rheum Dis. 2016;75:75-83

injsma JWJ, et al. Lancet. 2016;388:343-355

Brunekreef T, et al. Ann Rheum Dis. 2017;76:147-148

de Jong PH, et al Ann Rheum Dis. 2014:73:1331-1339

Dougados MR, et al. Rheumatol. 2014; 74:1233-1240

Emery P, 2017 et al. Ann Rheum Dis. 2017;76:96-104

torslev-Petersen K, et al. Ann Rheum Dis. 2014;73:654-6

Keystone EC, 2017 et al. Arthritis Rheum. 2017;60.

Keystone EC, 2017a et al. RMD Open. 2017;3

Ma MY, et al. J Rheumatol. 2014:41:1298-1303

Nam JL, a et al. Ann Rheum Dis. 2014a; $73: 75-85$

Nam JL, bet al. Ann Rheum Dis. 2014b;73:1027-1036

Smolen JC, 2014 et al. Lancet. 2014;383:321-332

molen JC, 2015 et al. Arthritis Res Ther. 2015

Steunebrink LMM, et al. Arthritis Res Ther. 2016;18:60

Stouten V, et al. Ann Rheum Dis. 2017:76:147

Takeuchi T, et al. Ann Rheum Dis. 2014;73:536-543

Ter Wee MM, et al. Ann Rheum Dis. 2015;74:1233-1240

Disclosure of Interests: Maxime Verhoeven: None declared, Paco Wels ing: None declared, Johannes WJ Bijlsma Grant/research support from: The department of the author who included patients (JWJB) in the U-ActEarly trial received reimbursements from Roche Nederland BV. JWJB reported grants and fees from Roche, AbbVie, Bristol-Myers Squibb, Merck Sharp \& Dohme, Pfizer, and UCB

University Medical Center Utrecht, Utrecht University, Consultant for: SUN Pharma, Speakers bureau: Lilly, Roche, Jacob M. van Laar Grant/ research support from: Genentech, Consultant for: F. Hoffmann-La Roche, Floris Lafeber Shareholder of: ArthroSave, Grant/research support from: FOREUM; Dutch Arthritis Society, Janneke Tekstra: None declared, Johannes W. G. Jacobs Grant/research support from: Roche, Consultant for: Roche

DOI: 10.1136/annrheumdis-2019-eular.3310

\section{THU0199 PHARMACOKINETICS, SAFETY AND TOLERABILITY OF SINGLE- AND MULTIPLE- DOSE ONCE-DAILY BARICITINIB IN CHINESE HEALTHY VOLUNTEERS - A RANDOMIZED PLACEBO-CONTROLLED STUDY}

Xia Zhao ${ }^{1}$, Christopher Payne ${ }^{2}$, Feng Wang ${ }^{3}$, Yiming Cui ${ }^{1} .{ }^{1}$ Peking University First Hospital, Beijing, China; ${ }^{2}$ Eli Lilly Research and Development, Windlesham, United Kingdom; ${ }^{3}$ Lilly Suzhou Pharmaceutical Co. Ltd. Shanghai Branch, Shanghai, China

Background: Baricitinib, an oral selective inhibitor of JAK1 and JAK2, is efficacious in patients with moderately to severely active rheumatoid arthritis and an inadequate response to methotrexate.

Objectives: To evaluate the pharmacokinetics (PK), safety and tolerability of baricitinib when given orally as single and multiple doses in healthy Chinese subjects.

Methods: This was a single-site, subject- and investigator-blinded, randomized, and placebo-controlled study (NCT02758613) conducted from 04 May 2016 to 25 July 2016. Eligible subjects were healthy Chinese adults ( $\geq 18$ years) with a body mass index of 19.0 to $24.0 \mathrm{~kg} / \mathrm{m}^{2}$ (inclusive). Subjects received a once daily dose (QD) of baricitinib 2, 4 or $10 \mathrm{mg}$ or placebo on Day 1 (single dose) and Days 4 through 10 for 7 consecutive days (multiple dose). Safety assessments were performed throughout the treatment period. Plasma PK samples were collected up to 48 hours after dosing on Days 1 and 10, with predose samples collected prior to dosing on Day 1 and Days 4 through 10. PK parameters were calculated using noncompartmental analysis.

Results: A total of 33 healthy volunteers were randomized and received at least 1 dose of study drug (placebo, $n=8$; baricitinib $2 \mathrm{mg}, \mathrm{n}=8$; baricitinib $4 \mathrm{mg}, \mathrm{n}=9$; baricitinib $10 \mathrm{mg}, \mathrm{n}=8$ ). All subjects were male and the mean age was 27.8 years. After both single- and multiple-dose administration, baricitinib 2 to $10 \mathrm{mg}$ was rapidly absorbed, reaching peak plasma concentrations within 0.5 to 1 hour (median). Plasma concentrations declined rapidly following attainment of peak concentrations, with a mean terminal half-life of 5.7 to 7.3 hours. Steady state plasma concentrations of baricitinib were achieved after the second day of QD dosing, with minimal accumulation of baricitinib in plasma (up to 10\%) during 7 days of QD oral doses of 2 to $10 \mathrm{mg}$ baricitinib. Single- and multipledose mean values for $\mathrm{AUC}_{(0-\infty)}$ and $\mathrm{C}_{\max }$ appeared to increase in an approximately dose-proportional manner across the dose range. Overall incidence of treatment-emergent AEs (TEAEs) is $33.3 \%$. All TEAEs reported were mild in severity, with no serious AEs reported and no subjects were withdrawn due to AEs. Although there was an apparent increase in the incidence of drug-related TEAEs over the 2 to $10 \mathrm{mg}$ dose range, the incidence of drug-related TEAEs at the $10 \mathrm{mg}$ dose was comparable to placebo.
Conclusion: The PK of baricitinib in Chinese healthy subjects were characterized by rapid absorption and elimination following single and multiple oral doses of up to $10 \mathrm{mg}$. Systemic exposure to baricitinib increased in an approximately dose-proportional manner following single and multiple doses. Single and multiple oral doses of daily-administered baricitinib up to $10 \mathrm{mg}$ were well tolerated by healthy Chinese subjects.

Disclosure of Interests: Xia Zhao: None declared, Christopher Payne Shareholder of: Eli Lilly and Company, Employee of: Eli Lilly and Company, Feng Wang Shareholder of: Eli Lilly and Company, Employee of: Eli Lilly and Company, Yiming Cui: None declared DOI: 10.1136/annrheumdis-2019-eular.4699

\section{THU0200 DRUG UTILISATION IN PEOPLE WITH EARLY RHEUMATOID ARTHRITIS IN THE UNITED KINGDOM}

Sarah Wood, Kimme Hyrich, Suzanne Verstappen, Douglas Steinke. The University of Manchester, Manchester, United Kingdom

Background: Despite a growing body of evidence in the pharmacological management of people with early rheumatoid arthritis (RA), there is still uncertainty concerning the most effective treatment regimen. Until 2018 guidance for England and Wales stated that everyone with early RA should receive combination therapy with 2 or more conventional synthetic disease modifying anti-rheumatic drugs (csDMARDs) in parallel. However, from epidemiological studies and anecdotal information, we know that this has not been the case in practice.

Objectives: (i) To determine the proportion of patients with new onset RA who start methotrexate (MTX) either alone or in combination with another csDMARD (ii) to describe which combinations are currently being prescribed and (iii) to describe the characteristics associated with the choice of initial RA treatment strategy

Methods: Consecutive patients with RA participating in the Rheumatoid Arthritis Medication Study (RAMS), a large UK study recruiting patients starting MTX for the first time, were eligible. Data on demographics, dis ease activity, comorbidity and all RA treatments are captured at baseline, 6 and 12 months following start of MTX. Analysis was limited to patients starting MTX within the first 2 years of symptom onset as part of their first treatment regimen for RA. Prevalent users of alternative csDMARDs, defined as use > 6 weeks prior to MTX start, were excluded. Participants were categorised as either starting MTX monotherapy or MTX in combination with another csDMARD (defined as started +/- 6 weeks of MTX start date). Baseline characteristics were compared between monotherapy and combination therapy groups using descriptive statistics.

Results: 1,374 patients with a mean (SD) age of 58 (14) years were included in this study; 64\% were female. At baseline, 996 (76\%) started MTX monotherapy and $316(24 \%)$ started MTX/csDMARD combination therapy (84\% with hydroxychloroquine and $16 \%$ with sulphasalazine) Patients starting combination therapy were younger $(p<0.005)$, with higher DAS28 scores $(p<0.005)$, higher HAQ scores $(p<0.05)$, lower MTX starting doses $(p<0.005)$, and shorter symptom duration $(p<0.05)$ compared with the monotherapy group.

Conclusion: These data show that despite national guidelines recommending combination csDMARD therapy in all patients diagnosed with RA, over three quarters of patients still started MTX monotherapy. People who were prescribed combination therapy had more severe disease sug gesting that prescribers do consider prognosis in their treatment decisions. A majority of patients starting combination therapy did so with hydroxychloroquine, despite a lack of evidence for the benefits of this particular combination in clinical practice.

Table 1. Baseline characteristics of the monotherapy and combination therapy groups

\begin{tabular}{lccc}
\hline Characteristic & $\begin{array}{c}\text { Monotherapy } \\
(\mathrm{n}=1,034)\end{array}$ & $\begin{array}{c}\text { Combination therapy } \\
(\mathrm{n}=340)\end{array}$ & $\begin{array}{c}\mathrm{P} \\
\text { value }\end{array}$ \\
\hline Age (years) & $61(50,70)$ & $57(46,67)$ & 0.001 \\
Female sex & $655(63)$ & $223(66)$ & 0.455 \\
Symptom duration (months) & $6.3(3.7,11.0)$ & $5.8(3.4,9.0)$ & 0.020 \\
HAQ score & $1.0(0.4,1.5)$ & $1.13(0.5,1.6)$ & 0.015 \\
DAS28 & $4.1(3.2,5.1)$ & $4.5(3.5,5.5)$ & 0.002 \\
Co-morbidities: $>=1$ & $468(45)$ & $130(38)$ & 0.023 \\
MTX starting dose (mg/ & $15(10,15)$ & $10(10,12.5)$ & 0.000 \\
week) & & & \\
\hline
\end{tabular}

Values are frequency (\%) or median (IQR)

Abbreviations: HAQ, health assessment questionnaire; DAS, disease activity score; MTX, methotrexate 
Disclosure of Interests: Sarah Wood Grant/research support from: Pfizer stipend received for MPhil in epidemiology in 2013, Employee of: Employed by GSK in 2012 as a pre-registration pharmacist, Kimme Hyrich Grant/research support from: Grants to institution: BMS, Pfizer, UCB, Suzanne Verstappen: None declared, Douglas Steinke: None declared

DOI: 10.1136/annrheumdis-2019-eular.4721

\section{THU0201 ANALYSIS OF GUT MICROBIOTA DIVERSITY IN PATIENTS WITH RHEUMATOID ARTHRITIS AND BENEFICIAL EFFECTS OF PROBIOTICS ON INTESTINAL FLORA}

Ming Yan ${ }^{1}$, Sheng-Xiao Zhang ${ }^{1}$, Chong Gao ${ }^{2}$, Xiao-Bin Zheng ${ }^{1}$, Lili Shang ${ }^{1}$, XuFang Yin ${ }^{1}$, Zhang Mingxing ${ }^{1}$, Jia Wang ${ }^{1}$, Ting Cheng ${ }^{1}$, Nalin Lai ${ }^{1}$, LI Xiao-Feng ${ }^{1}$. ${ }^{1}$ The Second Hospital of Shanxi Medical University, Department of Rheumatology, Taiyuan, China; ${ }^{2}$ Brigham and Women's Hospital, Harvard Medical School, Department of Pathology, Boston, United States of America

Background: Rheumatoid arthritis (RA) is a progressive, irreversible disease characterized by autoimmune imbalance. Recent studies have found that the number and type of intestinal flora in RA patients were changed (called disorders), which in turn leads to immune imbalance in RA patients. The breath test can indirectly assess the growth of bacteria in the intestine. This study aimed to investigate the intestinal flora imbalance in RA patients and the effect of probiotics on the intestinal flora of RA patients using methane and hydrogen breath test.

Objectives: To study the beneficial effects of probiotics on the intestinal flora of RA patients by detecting the concentrations of methane and hydrogen and the microbial populations in patients with rheumatoid arthritis (RA) before and after probiotic treatment.

Methods: The lactulose methane and hydrogen breath technique were used to detect and compare the concentration of methane and hydrogen in 36 patients with RA before and after probiotic treatment (mainly Bifidobacterium, Clostridium butyricum, Lactobacillus) for 3 weeks. At the same time, the 16SRNA V3 region in stool samples of 17 patients with RA was qualitatively analyzed by Roche/45 high-throughput sequencing platform and compared with 9 healthy adult stool samples.

Results: Compared with pre-intervention, after 3 weeks of probiotic treatment, the exhaled methane concentration was significantly increased in RA patients at 30 minutes, 60 minutes, and 90 minutes $(P<0.05)$. The sum of methane and hydrogen concentrations increased at 30 minutes $(P=0.041)$. There was no significant difference in hydrogen concentration before and after intervention $(P>0.05)$ (Table 1). Compared with healthy adults, the fecal diversity index (Shannon) of RA patients increased $(P<$ 0.05 ) and the species uniformity index (flatness) also increas.
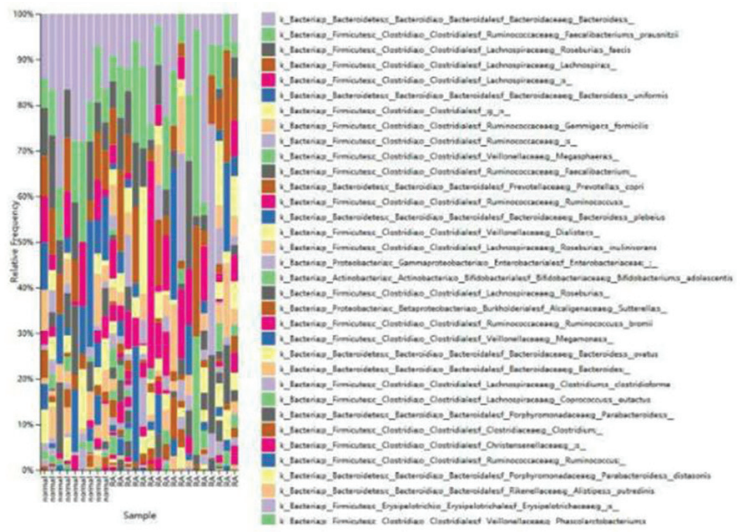

Figure 1: Compared with healthy subjects $(n=9)$, RA patients $(n=17)$ increased the fecal diversity index, $P<0.05$

\section{Abstract THU0201 - Figure 1}

Conclusion: Patients with RA had a significant difference in the structure of intestinal flora compared with that of healthy controls, which may represent perturbations of microbial communities and contribute to the pathogenesis and condition of RA. Probiotics can restore the composition of the gut microbiome and introduce beneficial functions to gut microbial communities through diet, probiotics or fecal transplantation, providing a promising prospect for clinical treatment of RA.

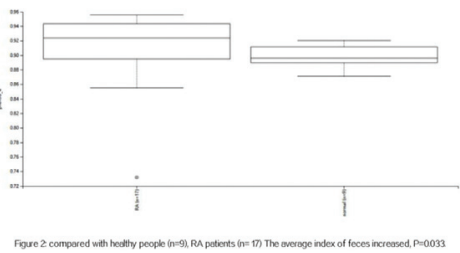

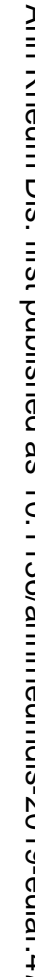
SLE, Sjögren's and APS - etiology, pathogenesis
and animal models

\begin{tabular}{|l|l|}
\hline THU0202 & INTERFERON STIMULATED GENE 15 PROTECTS \\
REGULATORY T CELL OF SYSTEMIC LUPUS \\
ERYTHEMATOSUS PATIENTS FROM INTERFERON \\
ALPHA-MEDIATED DEPLETION
\end{tabular}

Francesca Romana Spinelli, llenia Pacella, Silvia Piconese, Fulvia Ceccarelli, Francesca Miranda, Cristiano Alessandri, Vincenzo Barnaba, Guido Valesini, Fabrizio Conti. Sapienza University of Rome, Rome, Italy

Background: Data on $T$ regulatory ( $T$ reg) cells number and functions in patients with Systemic Lupus Erythematosus (SLE) are conflicting (1). Type I interferon (IFN) decreases Treg proliferation and induces Treg apoptosis (2). IFN stimulated gene 15 (ISG15) is an IFN-induced protein with a negative effect on IFN pathway.

Objectives: Aim of the study was to evaluate the effect of IFN alpha and ISG15 on Treg number and on STAT1 phosphorilation in patients with SLE. Methods: We recruited patients with SLE classified according to 1997 ACR criteria. We collected demographic and clinical data including disease duration, disease activity scored according to Systemic Lupus Erythematosus Disease Activity Index (SLEDAI) 2K. Peripheral blood mononuclear cells (PBMC) were isolated from whole blood samples. Intracellular phosphorilated STAT1 (pSTAT) levels were evaluated in Treg (CD4+FOXP3+CD127 low cells) and conventional $\mathrm{T}$ cell (Tconv, CD4 +FOXP3- cells) by multiparametric flow cytometry, ex vivo or after stimulation with recombinant IFN alpha. PBMC were cultured for 48 hours with anti-CD3 or anti-CD3 and IFN alpha; the frequency of Treg was analysed by multiparametric flow cytometry. ISG15 mRNA expression was evaluated by RT-PCR on PBMC from SLE patients. Data were expressed as mean \pm standard deviation or median (interquartile range(according) to the distribution; Mann-Whitney and Spearman tests were applied. P value $<0.05$ were considered statistically significant.

Results: We enrolled 21 SLE patients [F:M 20:1; mean age $45.2 \pm 12.9$ years; mean disease duration 157.5 \pm 103 months; median SLEDAI 2(4)] Overall, median baseline ISG15 mRNA expression was 0.06 (IQR 0.16); patients were divided into ISG15-high $(\mathrm{n}=9)$ and ISG15-low $(\mathrm{n}=12)$, according to the mean mRNA expression. Ex vivo, after short-term treatment with IFN alpha (15 and 25 minutes), we observed a significant increase in STAT1 phosphorilation compared to baseline both in Treg and Tconv $(p<0.00001)$, in ISG15-high and ISG15-low patients (Figure $1 \mathrm{~A}$ and B); however, high ISG15 levels protect both Treg and Tconv from STAT1 phosphorilation (Figure $1 \mathrm{C}$ ). After 48 of colture with IFN alpha, we observed a decrease in Treg and Tconv number, significantly greater in ISG15-low cells (Figure 1D). Treg frequency and ISG15 expression in Treg were higher in active vs inactive SLE patients [SLEDAI > 4 vs SLEDAI $<4] \quad(p<0.05)$.

Conclusion: ISG15 protects T conventional cells from STAT1 phosphorilation and induces resistance to $T$ regulatory cells depletion. The results of the study suggest that ISG15, a protein induced by IFN in the acute phase of the disease, exerts a negative feedback, allowing the recovery of $T$ reg. 